\title{
Medial auditory thalamus inactivation prevents acquisition and retention of eyeblink conditioning
}

\author{
Hunter E. Halverson, Amy Poremba, and John H. Freeman ${ }^{1}$ \\ Department of Psychology, University of lowa, lowa City, lowa 52242, USA
}

\begin{abstract}
The auditory conditioned stimulus (CS) pathway that is necessary for delay eyeblink conditioning was investigated using reversible inactivation of the medial auditory thalamic nuclei (MATN) consisting of the medial division of the medial geniculate (MGm), suprageniculate (SG), and posterior intralaminar nucleus (PIN). Rats were given saline or muscimol infusions into the MATN contralateral to the trained eye before each of four conditioning sessions with an auditory CS. Rats were then given four additional sessions without infusions to assess savings from the initial training. All rats were then given a retention test with a muscimol infusion followed by a recovery session. Muscimol infusions through cannula placements within $0.5 \mathrm{~mm}$ of the $\mathrm{MGm}$ prevented acquisition of eyeblink conditioned responses (CRs) and also blocked CR retention. Cannula placements more than $0.5 \mathrm{~mm}$ from the MATN did not completely block CR acquisition and had a partial effect on CR retention. The primary and secondary effects of MATN inactivation were examined with 2-deoxy-glucose (2-DG) autoradiography. Differences in 2-DG uptake in the auditory thalamus were consistent with the cannula placements and behavioral results. Differences in 2-DG uptake were found between groups in the ipsilateral auditory cortex, basilar pontine nuclei, and inferior colliculus. Results from this experiment indicate that the MATN contralateral to the trained eye and its projection to the pontine nuclei are necessary for acquisition and retention of eyeblink CRs to an auditory CS.
\end{abstract}

Pavlovian eyeblink conditioning is an ideal paradigm for identifying the necessary and sufficient neural structures underlying associative learning (Thompson 1976, 1983). Eyeblink conditioning is typically established by pairing a conditioned stimulus (CS) such as a tone or light with an unconditioned stimulus (US) that elicits the eyeblink reflex. An eyeblink conditioned response (CR) to the CS emerges over the course of training and occurs prior to the onset of the US. Permanent lesions, reversible inactivation, stimulation, unit recording, and neuroanatomy have been used to establish that the interpositus nucleus and cerebellar cortex are the necessary sites of learning-dependent plasticity underlying eyeblink conditioning (Thompson 2005). The basilar pontine nuclei are the proximal end of the CS pathway, which send mossy fiber input to the contralateral cerebellar cortex and interpositus nucleus (Steinmetz et al. 1986, 1987; Lewis et al. 1987; Knowlton and Thompson 1988; Steinmetz 1990; Steinmetz and Sengelaub 1992; Hesslow et al. 1999; Freeman and Rabinak 2004; Freeman et al. 2005).

Possible auditory inputs to the pontine nuclei that could provide auditory CS information have been proposed (Steinmetz et al. 1987; Steinmetz and Sengelaub 1992), but a systematic experimental analysis of these inputs has not been done. The ventral cochlear nucleus has a monosynaptic projection to the pontine nuclei in rats and rabbits (Steinmetz et al. 1987; Campolattaro et al. 2007), but this short-latency auditory pathway may not be sufficient to support eyeblink conditioning (Halverson and Freeman 2006). Neurons in the lateral pontine nuclei respond to auditory CSs with latencies between 3 and $7 \mathrm{msec}$, and a population of interpositus neurons shows a response latency of $<20$ msec to an auditory CS (Steinmetz et al. 1987; Berthier and Moore 1990; Steinmetz and Sengelaub 1992; Nicholson and Freeman 2002; Freeman and Muckler 2003). However, an ISI $>50$ msec is needed to establish conditioning, leaving the possibility that other auditory structures are critically involved in relaying auditory CS information to the pontine nuclei (Smith et al. 1969; Halverson

\footnotetext{
'Corresponding author.
}

E-mail john-freeman@uiowa.edu; fax (319) 335-0191.

Article is online at http://www.learnmem.org/cgi/doi/10.1101//m.1002508. and Freeman 2006). The auditory cortex also has a direct projection to the pontine nuclei, but decerebrations rostral to the red nucleus and complete decortication do not prevent acquisition or retention of auditory eyeblink conditioning (Oakley and Russell 1972, 1977; Mauk and Thompson 1987; Knowlton et al. 1993). Stimulation of other auditory system structures, including the superior olive, inferior colliculus (IC), and medial (MGm) and ventral (MGv) divisions of the medial geniculate, are effective CSs for eyeblink conditioning (Patterson 1970; Nowak et al. 1999; Campolattaro et al. 2007). The Campolattaro et al. (2007) study also showed that eyeblink CRs established using MGm stimulation as the CS are not dependent on thalamic feedback to the IC. The IC has a direct projection to the pons, and large IC lesions attenuate $\mathrm{CR}$ acquisition but do not block learning, providing evidence that other structures in the auditory system are sending information to the pontine nuclei (Kawamura 1975; Freeman et al. 2007).

Neural activity in the MGm shows CS- and CR-related facilitation during differential auditory trace eyeblink conditioning (O'Connor et al. 1997) and other Pavlovian conditioning procedures (Disterhoft and Olds 1972; Ryugo and Weinberger 1978; Supple Jr. and Kapp 1989; Edeline and Weinberger 1992; Lennartz and Weinberger 1992; McEchron et al. 1995). Complete neurotoxic lesions of the medial auditory thalamic nuclei (MATN) including the MGm, posterior intralaminar nucleus (PIN), and suprageniculate nucleus (SG) contralateral to the trained eye block acquisition of eyeblink conditioning to an auditory CS even with extended training (2000 trials), but spare conditioning to a light CS (Halverson and Freeman 2006). The effects of MATN lesions on retention of eyeblink conditioning have not been examined. The MATN also has a direct and exclusively ipsilateral projection to the medial and lateral pontine nuclei, which might be necessary for auditory conditioning (Campolattaro et al. 2007). This study examined the role of the MATN in acquisition and retention of auditory eyeblink conditioning using unilateral muscimol inactivation in rats. The direct and indirect metabolic effects of thalamic infusions of muscimol were assessed with 2-DG imaging. 


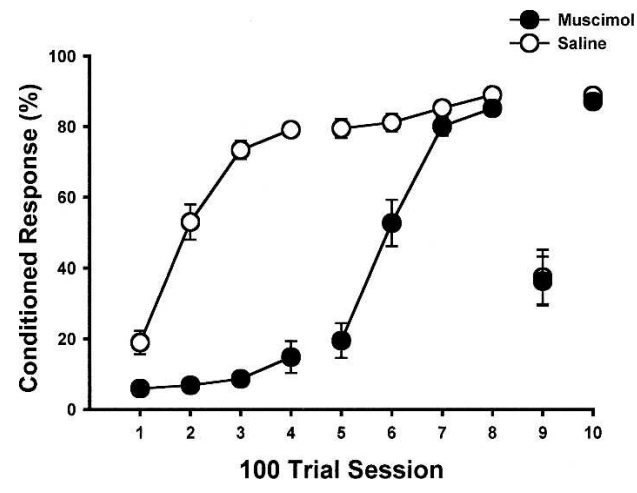

Figure 1. Mean $\pm S E$ conditioned response $(C R)$ percentage for rats given muscimol or saline infusions into the medial auditory thalamus (MATN) before sessions 1-4. Rats were given additional training without infusions during sessions 5-8. All rats were then given a final infusion of muscimol on session 9 , followed by a recovery session.

\section{Results}

Muscimol infusions into the MATN contralateral to the conditioned eye severely impaired acquisition of CRs during phase I (Fig. 1, sessions 1-4). Acquisition rate in the muscimol group during phase II (sessions 5-8), with no infusions, was identical to the phase I acquisition rate of the saline group, indicating that associative learning was specifically impaired in the muscimol group in the first phase of training. Both groups showed a substantial decrease in CR percentage during the muscimol retention test (session 9) and complete recovery of CRs during the subsequent recovery session (session 10). However, the muscimol retention test did not produce a complete loss of CRs.

Repeated-measures ANOVA on the CR percentage data for the muscimol and saline groups for the first two phases (sessions 1-8) revealed an interaction of the group, session, and phase factors $\left(F_{(3,87)}=91.546 ; p<0.0001\right)$. Post-hoc tests indicated that the saline group produced more CRs than the muscimol group on sessions 1-6 (all comparisons, $p<0.05$ ). The magnitude of the difference increased across sessions $1-4$ and decreased across sessions 5 and 6. Repeated-measures ANOVA on the CR percentage data for the muscimol and saline groups for the retention test (sessions 8-10) revealed a main effect of the session factor $\left(F_{(2,58)}=89.736\right.$; $p<0.0001)$. Post-hoc tests indicated that all rats showed fewer CRs on the muscimol retention test (session 9) than on sessions 8 and $10(p<0.05)$.

Figure 2 shows cannula placements in coronal sections of the auditory thalamus. The placements for the saline and muscimol groups were in or within 0.5 $\mathrm{mm}$ of the MGm except for seven medial placements in the anterior pretectal nucleus, three lateral placements in the ventral or dorsal divisions of the medial geniculate nucleus, and one placement ventral to the PIN.

The purpose of the muscimol retention test was to examine the effects of MATN inactivation on CR retention in both the muscimol and saline groups. Consistent differences were observed in the effect of muscimol during the retention test between rats with cannula placements within $0.5 \mathrm{~mm}$ or directly in the MGm, and rats with placements out- side that range. Based on the cannula proximity to the MGm, rats were put into one of four groups for additional analyses: muscimol hit $(n=7)$, muscimol miss $(n=7)$, saline hit $(n=9)$, or saline miss $(n=8)$. Rats with cannulae in or within $0.5 \mathrm{~mm}$ of the MGm were put into the muscimol hit and saline hit groups. Rats with cannula outside $0.5 \mathrm{~mm}$ of the MGm were put into the muscimol miss and saline miss groups. Rats in the muscimol hit group showed no evidence of acquisition in phase I, no savings in phase II $\left(F_{(9,81)}=40.244 ; p<0.0001\right)$, and a severe impairment during the muscimol retention test $\left(F_{(6,54)}=56.167 ; p<0.0001\right)$ (Fig. $3 \mathrm{~A})$. An equally severe deficit was seen in the saline hit group during the retention test. Rats in the muscimol miss group showed some evidence of acquisition in phase I, more rapid acquisition in phase II, and a moderate impairment during the muscimol retention test (Fig. 3B).

Analysis of 2-DG autoradiographs taken from rats given exposure to the CS were used to qualitatively and quantitatively examine the metabolic effects of inactivating the MATN contralateral to the trained eye. Qualitative analysis of the muscimol hit $(n=6)$, muscimol miss $(n=4)$, and saline hit $(n=4)$ groups indicated that uptake of 2-DG in the right MATN was reduced substantially in the animals given muscimol. Rats in the saline hit group displayed robust metabolic activity in the MATN (Fig. 4). The muscimol hit group showed a severe reduction in the amount of 2-DG uptake in the right MATN (Fig. 4). The muscimol miss group also showed a reduction in 2-DG uptake in the right MATN, but to a lesser extent than the muscimol hit group (Fig. 4). Region-of-interest readings were taken from the entire auditory cortex, inferior colliculus, basal pontine nuclei, hippocampus, interpositus nucleus, and lobule HVI and anterior lobe of the cerebellum (Table 1; Fig. 5) to determine the secondary metabolic effects of muscimol inactivation of the MATN on the right side. A reduction in 2-DG uptake (in nanocuries per gram, $\mathrm{nCi} / \mathrm{g}$ ) for the muscimol hit and miss groups, compared to the saline hit group, was found in the right auditory cortex, right inferior colliculus, right hippocampus, and right pontine nuclei. Uptake of 2-DG was reduced on the right side compared to the left within the muscimol hit and miss groups for the auditory cortex, inferior colliculus, and pontine nuclei.

Analysis was done on the 2-DG data to investigate activity differences in each structure between the muscimol and saline
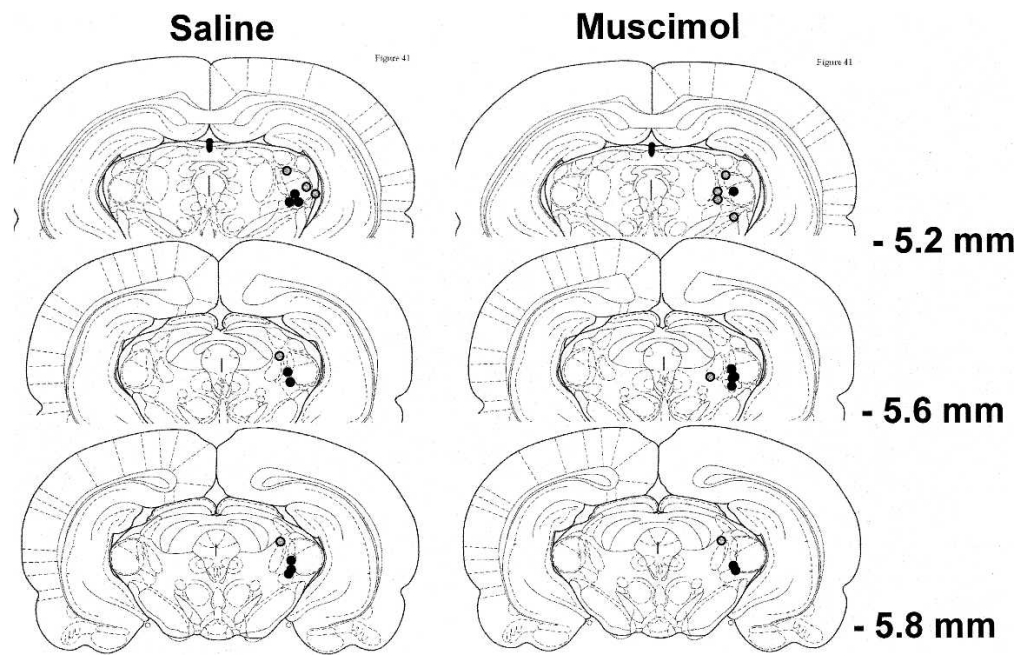

Figure 2. Coronal sections of the rat auditory thalamus depicting the cannula placements for the muscimol and saline groups. Cannula placements classified as hits (black dots) were within $0.5 \mathrm{~mm}$ of the MGm. Cannula placements outside of this range (gray dots) were classified as misses. Adapted with permission from Paxinos and Watson (1998). 

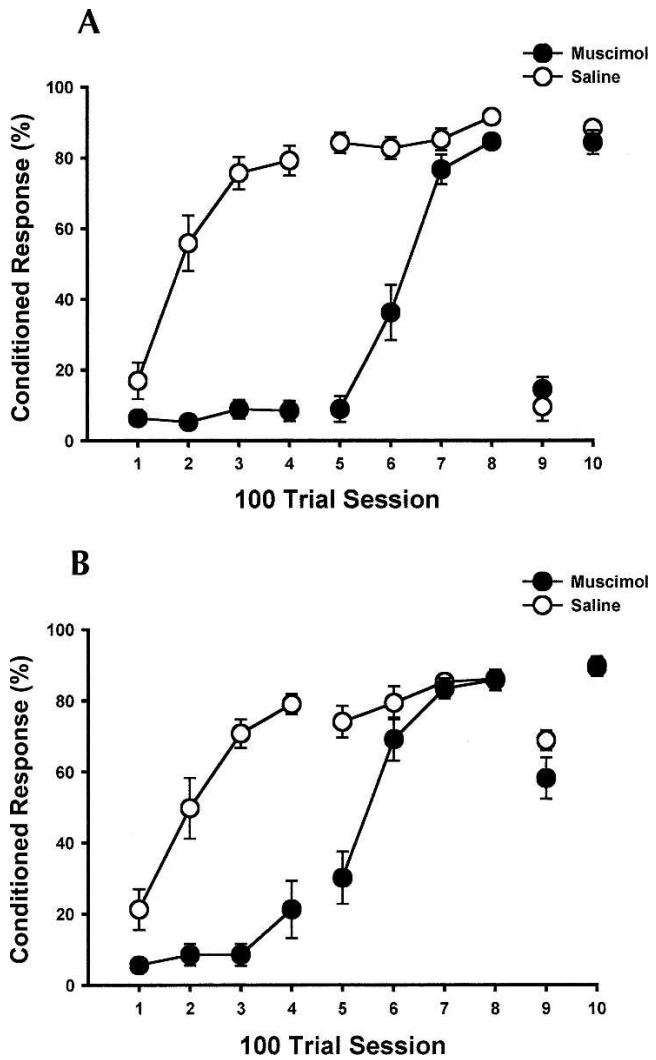

Figure 3. Mean $\pm \mathrm{SE}$ conditioned response $(\mathrm{CR})$ percentage for rats given muscimol or saline separated into $(A)$ hit and $(B)$ miss groups, based on cannula placement. (B) Rats in the miss group show evidence of learning on session 4 and savings on sessions 5 and 6 relative to the hit group. (B) Rats in the miss group show a partial impairment on a muscimol retention test (session 9). (A) Rats in the hit group show a complete impairment during the muscimol retention test.

groups, and also to identify activity differences between the left and right side of each structure between groups. Repeatedmeasures ANOVA on the normalized region-of-interest 2-DG readings revealed an interaction of the group and side factors for the pontine nuclei $\left(F_{(2,7)}=11.499 ; p<0.01\right)$, hippocampus $\left(F_{(2,10)}=7.312 ; p<0.05\right)$, inferior colliculus $\left(F_{(2,9)}=16.325\right.$; $p<0.001)$, and auditory cortex $\left(F_{(2,11)}=12.632 ; p<0.001\right)$. Posthoc tests indicated that the saline hit group showed more 2-DG uptake in the right basal pontine nuclei, right hippocampus, right inferior colliculus, and right auditory cortex than the muscimol hit and miss groups $(p<0.05)$. There was also more $2-\mathrm{DG}$ uptake on the left side of the pontine nuclei and auditory cortex than the right side for the muscimol hit and miss groups $(p<0.05)$ (Fig. 5). The magnitude of the difference was larger in the hit group in the pontine nuclei and larger for the miss group in the auditory cortex. Only the muscimol hit group showed a reduction in the left inferior colliculus compared to the right within groups $(p<0.05)$.

\section{Discussion}

Acquisition and retention of auditory delay eyeblink conditioning were severely impaired by muscimol inactivation of the MATN contralateral to the conditioned eye. Rats with accurately placed thalamic cannulae (muscimol hit group) showed no evidence of associative learning during acquisition (Fig. 3A, sessions 1-4) and no savings during initial retraining (Fig. 3A, sessions 5-8) following muscimol infusions. A muscimol retention test completely blocked CRs in rats with accurately placed cannulae that were given either saline or muscimol during acquisition (Fig. 3A, session 9). Rats with slightly misplaced cannulae (muscimol miss group) were also severely impaired during acquisition but showed savings during initial retraining (Fig. 3B, sessions 5-8). A muscimol retention test partially impaired CRs in both muscimol miss and saline miss groups (Fig. 3B, session 9).

Uptake of 2-DG in the MATN contralateral to the conditioned eye was substantially reduced relative to the ipsilateral side in the muscimol hit group (Fig. 4). The difference in 2-DG uptake between the contralateral and ipsilateral MATN in the muscimol miss group was less pronounced (Fig. 4). The difference in the magnitude of inactivation in the MATN between the hit and miss groups could account for the behavioral differences observed between these two groups during initial retraining and the retention test. Uptake of 2-DG in the saline hit group did not differ between the left and right sides.

A relatively large infusion volume was used in this experiment $(1.0 \mu \mathrm{L})$ to target the entire MATN (MGm, PIN, SG) with a single infusion. A previous neurotoxic lesion study found that complete neuron loss in the MATN was necessary to block conditioning to an auditory CS. Sparing of any of these nuclei did not produce an impairment in acquisition (Halverson and Freeman 2006). Despite the relatively large infusion volume, structures in the brainstem and cerebellum important for delay eyeblink conditioning such as the facial motor nuclei, inferior olive, pontine nuclei, interpositus nucleus, and red nucleus were not directly inactivated by muscimol. Slight variations in cannula placement and inactivation around the MATN produced consistent behavioral differences in both acquisition and retention of CRs in the current experiment, suggesting that inactivation of the MATN, and not other structures, is necessary to block auditory eyeblink conditioning.

Quantitative analysis of 2-DG uptake between the saline and muscimol groups revealed significant secondary effects of MATN inactivation in other neural structures. The MATN have direct unilateral projections to the basal pontine nuclei, IC, and auditory cortex (Kawamura 1975; Winer et al. 1999, 2001; Donishi et al. 2006; Campolattaro et al. 2007), and all of these structures showed a reduction in 2-DG uptake in the hemisphere ipsilateral to the muscimol infusion (Table 1; Fig. 5). The IC and auditory cortex also directly project unilaterally to the pontine nuclei, so the decrease in metabolic activity seen in these structures could have contributed to the decrease in 2-DG uptake in the pons, in addition to the reduction in input from the thalamus (Kawamura 1975; Knowlton et al. 1993). Muscimol-induced differences in 2-DG uptake were also observed between the left and right side within groups for the basal pontine nuclei, IC, and auditory cortex (Table 1; Fig. 4). The neural projections between the IC, MG, and auditory cortex and from auditory structures to the pontine are primarily unilateral, suggesting that a decrease in metabolic activity on one side of the upper auditory system would be an expected secondary consequence of MATN inactivation. There was also a slight nonsignificant reduction of 2-DG uptake in the IC, auditory cortex, and basal pontine nuclei contralateral to the inactivated side (Table 1; Fig. 5). This reduction in 2-DG uptake was probably due to decreased commissural input from the inactivated side. The reduction in 2-DG uptake within the pontine nuclei in the muscimol groups indicates that auditory information from the MATN to the pontine was blocked. Accordingly, auditory-evoked neuronal activity in the pontine nuclei should be abolished by thalamic inactivation.

No significant differences in 2-DG uptake were found in the cerebellum either between or within groups (Table 1). Inactivation of the right MATN did result in decreased metabolic activity in the ipsilateral pons, and this decrease in activity was likely due 


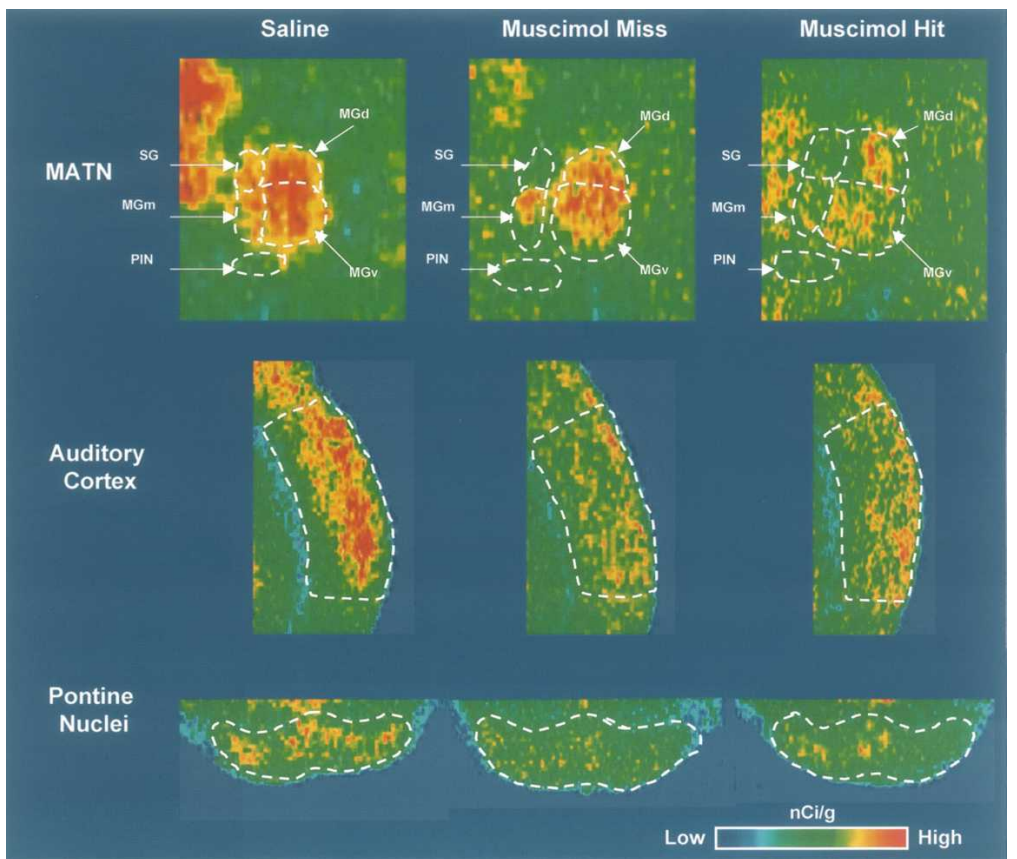

Figure 4. Autoradiographs showing uptake of 2-DG radiolabeled with ${ }^{14} \mathrm{C}$ (red indicating strongest 2-DG uptake). (Dotted lines) Boundaries of the medial auditory thalamic nuclei (MATN), auditory cortex, and basal pontine nuclei. Boundaries were drawn based on the same nissl-stained sections. (Upper row) Representative MATN sections showing activity in the auditory thalamus in the muscimo hit, muscimol miss, and saline groups. (Middle row) Auditory cortex and (lower row) pontine sections show secondary inactivation after ipsilateral MATN saline or muscimol infusions.

to reduced auditory input. Sensory inputs from visual, somatosensory, and cortical areas were probably still being relayed into the cerebellum through the pontine mossy fiber system during MATN inactivation (Glickstein et al. 1980; Schmahmann and Pandya 1989, 1993). Other inputs including the lateral reticular nucleus, which is also a major source of mossy fibers that primarily synapse on the interpositus nucleus, could also have masked small differences in cerebellar metabolic activity due to MATN inactivation (Kunzle 1975; Bloedel and Courville 1981; Knowlton et al. 1988). The use of CS-alone trials during 2-DG uptake also caused some extinction of CRs and probably produced a corresponding decrease in neural activity in the interpositus nucleus (Gould and Steinmetz 1996), making metabolic activity differences more difficult to detect. Metabolic mapping studies using extinction of fear conditioning have also failed to find tone-evoked metabolic activity differences in the lateral and basal lateral amygdala, in contrast to the clear differences observed during acquisition of fear related CRs (Barrett et al. 2003).

Although the hippocampus was partially inactivated with muscimol infusions into the thalamus, it is unlikely that the impairments in acquisition and retention were due to hippocampal inactivation. The amount of hippocampal inactivation was minimal relative to the thalamic inactivation. Moreover, the hippocampal inactivation did not differ between the hit and miss groups. These findings coupled with the previous studies that showed no deficits in delay conditioning with large bilateral hippocampal lesions indicate that the incidental hippocampal inactivation in the present study played little or no role in the conditioning deficits (Schmaltz and Theios 1972; Solomon and Moore 1975).

This study provides additional evidence that the MATN contralateral to the trained eye is necessary for delay eyeblink conditioning to an auditory CS (Halverson and Freeman 2006). How- ever, the specific nature of the role of the MATN in eyeblink conditioning is unknown. The most likely possibility is that auditory CS information is passively relayed from the MATN into the cerebellum through the direct projection to the pontine nuclei (Campolattaro et al. 2007). The MATN may, therefore, be the critical sensory relay of auditory information for delay eyeblink conditioning to a tone CS. Alternatively, there could be learning-related plasticity in the MATN that facilitates cerebellar plasticity by boosting auditory input to the pontine nuclei. The MATN is a potential location of learning-related plasticity as neurons within these nuclei respond to auditory CSs as well as aversive USs and show selective retuning of their receptive fields to reinforced tones (Love and Scott 1969; Edeline and Weinberger 1992; Lennartz and Weinberger 1992; Bordi and LeDoux 1994). Studies using differential conditioning paradigms including trace eyeblink conditioning have also found evidence for learningrelated plasticity in the MATN (Gabriel et al. 1975; Ryugo and Weinberger 1978; Birt and Olds 1981; Supple Jr. and Kapp 1989; O'Connor et al. 1997; Poremba and Gabriel 2001). However, neuronal activity in the auditory thalamus does not differ between trials with and without CRs when using a threshold-level auditory CS, suggesting that MATN plastic-

Table 1. Mean and standard error of 2-DG $(\mathrm{nCi} / \mathrm{g})$ uptake

\begin{tabular}{|c|c|c|c|c|}
\hline \multirow[b]{2}{*}{ Structure group } & \multicolumn{2}{|c|}{ Left } & \multicolumn{2}{|c|}{ Right } \\
\hline & Mean & SE & Mean & SE \\
\hline \multicolumn{5}{|l|}{ IC } \\
\hline Muscimol hit & 266.9 & 20.4 & $226.9^{\mathrm{a}}$ & 21.0 \\
\hline Muscimol miss & 257.3 & 27.5 & $240.5^{b}$ & 25.0 \\
\hline Saline & 258.6 & 28.1 & 264.3 & 30.6 \\
\hline \multicolumn{5}{|l|}{ Hippocampus } \\
\hline Muscimol hit & 138.7 & 13.9 & $117.3^{b}$ & 12.3 \\
\hline Muscimol miss & 125.5 & 10.0 & $103.9^{\mathrm{b}}$ & 10.4 \\
\hline Saline & 150.0 & 24.8 & 146.6 & 29.1 \\
\hline \multicolumn{5}{|l|}{ Interpositus } \\
\hline Muscimol hit & 198.6 & 34.5 & 214.8 & 38.3 \\
\hline Muscimol miss & 193.7 & 26.9 & 191.7 & 25.9 \\
\hline Saline & 222.2 & 36.7 & 218.0 & 40.2 \\
\hline \multicolumn{5}{|l|}{ Anterior lobe } \\
\hline Muscimol hit & 155.4 & 25.0 & 141.2 & 25.2 \\
\hline Muscimol miss & 154.2 & 23.7 & 154.0 & 29.4 \\
\hline Saline & 199.3 & 36.0 & 198.1 & 39.2 \\
\hline \multicolumn{5}{|l|}{ Lobule HVI } \\
\hline Muscimol hit & 114.9 & 22.7 & 108.1 & 23.8 \\
\hline Muscimol miss & 106.1 & 22.1 & 105.5 & 27.2 \\
\hline Saline & 135.1 & 30.5 & 135.1 & 32.5 \\
\hline
\end{tabular}

andicates significant differences $(p<0.05)$ between right and left side within groups, and between the right side of the muscimol and saline groups.

IIndicates significant differences $(p<0.05)$ between the right side of the muscimol and saline groups. 

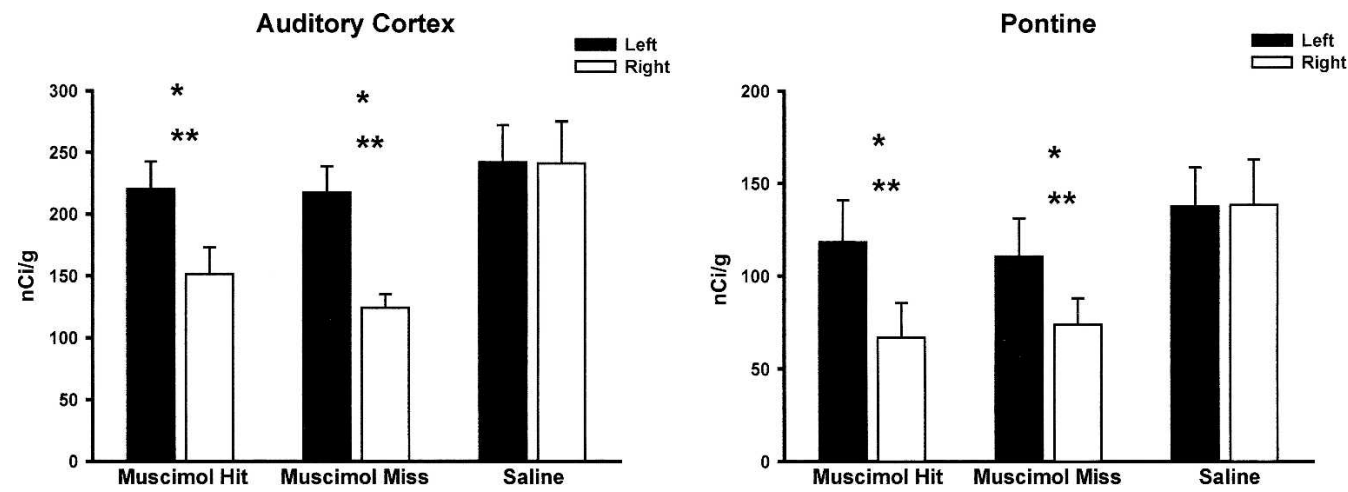

Figure 5. Mean $\pm \mathrm{SE} \mathrm{nCi/g}$ of 2-DG uptake in the left and right side of the auditory cortex and basal pontine nuclei for the muscimol hit, muscimol miss, and saline groups. (*) Significant differences $(p<0.05)$ between the muscimol groups and the saline group; $\left(^{* *}\right)$ significant differences $(p<0.05)$ between the right and left side within groups.

ity does not play a role in eyeblink conditioning (Kettner and Thompson 1985).

The results of the present study provide evidence that the medial auditory thalamic nuclei contralateral to the trained eye are necessary for acquisition and retention of delay eyeblink conditioning to an auditory CS in rats. The short-latency pathway from the cochlear nucleus to the pontine nuclei was intact during thalamic inactivation but was not sufficient for eyeblink conditioning with an auditory CS. It is possible, however, that the cochlear nucleus projection to the pontine nuclei is necessary for eyeblink conditioning in rabbits. The direct cochlear nucleus to pontine pathway in rabbits was suggested as a possible CS pathway based on auditory response latencies to peripheral stimuli in the pontine nuclei (Steinmetz et al. 1987; Steinmetz and Sengelaub 1992). Additional studies are needed in rabbits to determine whether species differences are present in auditory inputs to the pontine that are necessary for delay eyeblink conditioning. Our current hypothesis is that the necessary auditory CS pathway for eyeblink conditioning includes converging auditory inputs to the MATN and the direct unilateral thalamic projection to the pontine nuclei (Fig. 6). Current studies are determining whether information from the MATN must be relayed directly through the basilar pontine nuclei during eyeblink conditioning.

\section{Materials and Methods}

\section{Subjects}

The subjects were 31 male Long-Evans rats (250-400 g). The rats were housed in the animal colony in Spence Laboratories of Psychology at the University of Iowa (Iowa City, IA). All rats were maintained on a 12-h light/dark cycle and given ad libitum access to food and water.

\section{Surgery}

One week before training, rats were removed from their home cages and anesthetized with an intraperitoneal injection of sodium pentobarbital $(80 \mathrm{mg} / \mathrm{kg})$. The rats were given injections of atropine sulfate $(0.45 \mathrm{mg} / \mathrm{kg})$ to reduce respiratory tract secretions and excess salivation during anesthesia. At the onset of anesthesia, the rats were fitted with differential electromyograph (EMG) electrodes (stainless steel) that were implanted into the upper-left orbicularis oculi muscle. The reference electrode was a silver wire attached to a stainless steel skull screw. The EMG electrode leads terminated in gold pins in a plastic connecter. A bipolar stimulating electrode (Plastics One) for delivering the shock US was implanted subdermally, caudal to the left eye. A 23-gauge guide cannula was implanted $1.0 \mathrm{~mm}$ dorsal to the right medial division of the medial geniculate nucleus. A 30-gauge stylet was inserted into the guide cannula and extended $1.0 \mathrm{~mm}$ from the end of the guide. The stereotaxic coordinates taken from bregma were $5.5 \mathrm{~mm}$ posterior, $3.1 \mathrm{~mm}$ lateral, and $6.3 \mathrm{~mm}$ ventral to the skull surface. The plastic connector housing the EMG electrode leads, the bipolar stimulating electrode, the guide cannula, and three skull screws were secured to the skull with Osteobond copolymer bone cement (Zimmer). Animals were maintained on $3.0 \%$ Sulfatrim (Hi-Tech Pharmacal Co.) in water for $4 \mathrm{~d}$ after surgery.

\section{Muscimol infusion procedure}

Before the muscimol infusions, the stylet was removed from the guide cannula and replaced with a 30-gauge infusion cannula that extended $0.5 \mathrm{~mm}$ beyond the guide cannula. The infusion cannula was connected to polyethylene tubing (PE 10; 110-120 $\mathrm{cm}$ ), which was connected to a $10-\mu \mathrm{L}$ gas tight syringe (Hamilton). The syringe was placed in an infusion pump (Harvard Apparatus), and $1.0 \mu \mathrm{L}$ of muscimol (10 nmol, $\mathrm{pH} 7.4)$ was infused over $2 \mathrm{~min}$ at a rate of $30 \mu \mathrm{L} / \mathrm{h}$. The large infusion volume $(1.0$ $\mu \mathrm{L}$ ) was used to target all the nuclei of the MATN (MGm, PIN, and SG) with a single infusion. After the infusion, the tubing connected to the infusion cannula was cut and sealed with candle wax. The infusion cannula remained in place for the duration of the experimental session and was replaced by the stylet after each session.

\section{Apparatus}

The conditioning apparatus consisted of four small-animal sound-attenuating chambers (BRS/LVE). Within each soundattenuating chamber was a small-animal operant chamber (BRS/ LVE) in which the rats were kept during conditioning. One wall

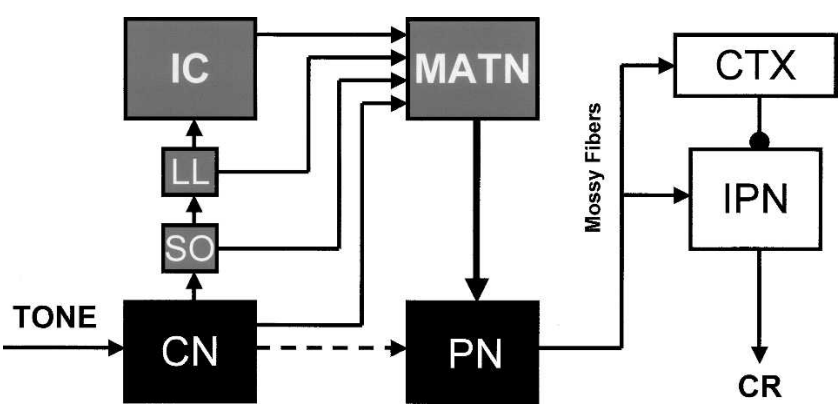

Figure 6. The auditory CS pathway for delay eyeblink conditioning Initially, tone information comes into the cochlear nucleus (CN). The $(\mathrm{CN})$, superior olive (SO), nucleus of the lateral leminiscus ( $\mathrm{LL})$, and inferior colliculus (IC) then converge onto the medial auditory thalamic nuclei (MATN). Auditory information is conveyed through a direct projection to the basal pontine nuclei (PN) from MATN and possibly the $\mathrm{CN}$. The PN sends mossy fiber projections into the cerebellum that synapse onto the cerebellar cortex (CTX) and interpositus nucleus (IPN). Finally, the IPN sends output for CR expression. 
of the operant chamber was fitted with two speakers. An exhaust fan on one of the walls provided a $65-\mathrm{dB}$ masking noise. The CS used in training was a $2000-\mathrm{Hz}$ pure tone $(85 \mathrm{~dB}$; range in conditioning chamber, 83-87 dB). The electrode leads from the rat's headstage were connected to peripheral equipment by lightweight cables that allowed the rat to move freely during conditioning. A desktop computer was connected to the peripheral equipment. Computer software controlled the delivery of stimuli and the recording of eyelid EMG activity (JSA Designs). The shock US was delivered through a stimulus isolator (model 365A; World Precision Instruments). The eyelid EMG activity was amplified differentially (gain, 2000; sampling rate, $250 \mathrm{~Hz}$ ), filtered $(500-5000 \mathrm{~Hz})$, and integrated (time constant, $20 \mathrm{msec})$. The intensity of the shock US was set at two times threshold for eliciting a discrete eyeblink (range of final current intensity, 2-4 mA; 60 $\mathrm{Hz}$; constant current).

\section{Conditioning procedure}

The rats were allowed to adapt to the training environment for 5 min before each training session. The rats were given saline $(n=17)$ or muscimol $(n=14)$ infusions into the MATN 30 min before each of four sessions of delay eyeblink conditioning (100 trials per day; pseudorandom distribution of intertrial intervals between 18 and $42 \mathrm{sec}$ that averaged $30 \mathrm{sec}$ ), in which a $400-\mathrm{msec}$ tone CS co-terminated with a 25-msec shock US, yielding an interstimulus interval of $375 \mathrm{msec}$. Each of the first four sessions was separated by $3 \mathrm{~d}$ to prevent tolerance to muscimol. Following the four infusion sessions, all rats were given four daily sessions without infusions to examine the amount of learning from the initial training. All rats then received a final infusion of muscimol followed by a recovery session. Daily training sessions consisted of 10 blocks of nine paired CS-US presentations followed by a CS alone trial. The values relayed to the computer software from the EMG integrator were units representing voltage values of integrated EMG activity. The CR threshold was set at $0.4 \mathrm{~V}$ above the amplified and integrated EMG activity at baseline. The EMG baseline was usually zero. Integrated EMG responses exceeding the threshold value during the first $80 \mathrm{msec}$ of the CS period were considered startle responses to the tone CS; responses that exceeded the threshold value during the last 295 msec of the CS-US interval were considered CRs; responses that crossed the threshold after US onset were defined as URs.

\section{Histology}

After training, some of the rats $(n=17)$ were euthanized with a lethal injection of sodium pentobarbital $(150 \mathrm{mg} / \mathrm{kg})$ and transcardially perfused with $\sim 100 \mathrm{~mL}$ of physiological saline followed by $\sim 300 \mathrm{~mL}$ of $10 \%$ neutral buffered formalin (Surgipath). After perfusion, the brains were post-fixed in the same fixative for a minimum of $24 \mathrm{~h}$, cryo-protected in a 30\% sucrose in formalin solution, and subsequently sectioned at $50 \mu \mathrm{m}$ with a sliding microtome. Sections were then stained with thionin. The location of the cannula placements was then verified using a light microscope (Leica DMLS) and a stereotaxic brain atlas (Paxinos and Watson 1998).

\section{2-DG procedure and analysis}

After training, some of the rats were given saline $(n=4)$ or muscimol $(n=10)$ infusions into the MATN 30 min before exposure to the tone CS used in training. Twenty minutes after the infusion, each rat received an intraperitoneal injection of a glucose analog, 2-DG radiolabeled with ${ }^{14} \mathrm{C}(20 \mu \mathrm{Ci} / 100 \mathrm{~g}$ of body weight; American Radiolabeled Chemicals) in $0.3 \mathrm{~mL}$ of sterile saline. After the injection, the rats were put into the training chamber and presented with $60 \mathrm{CS}$-alone trials during the $45 \mathrm{~min}$ of 2-DG uptake. Rats were then decapitated, and the brains were quickly removed, frozen at $-37^{\circ} \mathrm{C}$, and stored at $-80^{\circ} \mathrm{C}$. Brains were sectioned on a cryostat at $40 \mu \mathrm{m}$, and every section was kept. Sections were quick-dried on glass coverslips, glued on poster board, and exposed to ${ }^{14} \mathrm{C}$ PhosphorImaging screens for $36 \mathrm{~h}$ along with ${ }^{14} \mathrm{C}$ standards (Amersham Biosciences). The screens were read and digitized by a PhosphorImaging system
(Cyclone Storage Phosphor System; PerkinElmer Life Sciences). Individual calibration curves were calculated based on absolute gray levels of the ${ }^{14} \mathrm{C}$ standards on each film. Subsequent densitometric measures were then automatically converted to units of nanocuries per gram (nCi/g). Right versus left metabolic activity averages were then taken using region-of-interest measurements (ImageJ; NIH) from the entire auditory cortex (average readings per animal =82), inferior colliculus (46), hippocampus (111), pontine nucleus (44), interpositus nucleus (28), anterior lobe (32), and HVI (34) of the cerebellar cortex. Most of these structures were selected because of their direct neural connection to the MATN or contribution in acquiring and expressing the behavioral CR. The hippocampus was selected to assess the primary spread of inactivation from the site of muscimol infusion. The auditory thalamus (MGm, MGv, MGd) projects heavily to the ipsilateral auditory cortex, thus decreased metabolic activity in the ipsilateral auditory cortex was an expected result of MATN inactivation (Winer et al. 1999, 2001; Donishi et al. 2006). The inferior colliculus has primarily ipsilateral reciprocal projections with the auditory thalamus and also projects directly to the ipsilateral pontine nuclei, so decreased metabolic activity in the ipsilateral inferior colliculus was an expected result of MATN inactivation (Kawamura 1975). The MATN project directly to the ipsilateral basilar pons also, so decreased metabolic activity in the ipsilateral pons was also an expected result of MATN inactivation (Campolattaro et al. 2007). The pontine nuclei are the primary source of mossy fiber input into the contralateral cerebellum and project directly to the contralateral cerebellar cortex and to a lesser extent to the interpositus nucleus (Bloedel and Courville 1981; Brodal 1981; Steinmetz and Sengelaub 1992). Decreased cerebellar metabolic activity could, therefore, be a downstream effect from MATN inactivation. All readings were normalized using samples taken from the white matter; once normalized, all readings for each structure for each animal were averaged to compare $\mathrm{L} / \mathrm{R}$ differences across animals. Readings were not taken from the MATN because of the severe reduction in 2-DG uptake, which made identification of the nuclei difficult.

\section{Acknowledgments}

This work was supported by grants from the NIMH (MH080005) and NINDS (NS038890).

\section{References}

Barrett, D., Shumake, J., Jones, D., and Gonzalez-Lima, F. 2003. Metabolic mapping of mouse brain activity after extinction of a conditioned emotional response. J. Neurosci. 23: 5740-5749.

Berthier, N.E. and Moore J.W. 1990. Activity of deep cerebellar nuclear cells during classical conditioning of nictitating membrane extension in rabbits. Exp. Brain Res. 83: 44-54.

Birt, D. and Olds, M. 1981. Associative response changes in lateral midbrain tegmentum and medial geniculate during differential appetitive conditioning. J. Neurophysiol. 46: 1039-1055.

Bloedel, J.R. and Courville, L. 1981. Cerebellar afferent systems. In Handbook of physiology, Section I: The nervous system (eds. J.M. Brookhart et al.), Vol. II, pp. 735-830. Williams and Wilkins, Baltimore, MD.

Bordi, F. and LeDoux, J.E. 1994. Response properties of single units in areas of rat auditory thalamus that project to the amygdala. Exp. Brain Res. 98: 275-286.

Brodal, A. 1981. Neurological anatomy. Oxford University Press, New York.

Campolattaro, M.M., Halverson, H.E., and Freeman, J.H. 2007. Medial auditory thalamic stimulation as a conditioned stimulus for eyeblink conditioning in rats. Learn. Mem. 14: 152-159.

Disterhoft, J.F. and Olds, J. 1972. Differential development of conditioned unit changes in thalamus and cortex of rat. $J$. Neurophysiol. 35: 665-679.

Donishi, T., Kimura, A., Okamoto, K., and Tamai, Y. 2006. "Ventral" area in the rat auditory cortex: A major auditory field connected with the dorsal division of the medial geniculate body. Neuroscience 141: $1553-1567$.

Edeline, J.M. and Weinberger, N.M. 1992. Associative retuning in the thalamic source of input to the amygdala and auditory cortex: Receptive field plasticity in the medial division of the medial geniculate body. Behav. Neurosci. 106: 81-105. 
Freeman Jr., J.H. and Muckler, A.S. 2003. Developmental changes in eyeblink conditioning and neuronal activity in the pontine nuclei. Learn. Mem. 10: 337-345.

Freeman Jr., J.H. and Rabinak, C.A. 2004. Eyeblink conditioning in rats using pontine stimulation as a conditioned stimulus. Integr. Physiol. Behav. Sci. 39: 180-191.

Freeman Jr., J.H., Rabinak, C.A., and Campolattaro, M.M. 2005. Pontine stimulation overcomes developmental limitations in the neural mechanisms of eyeblink conditioning. Learn. Mem. 12: 255-259.

Freeman, J.H., Halverson, H.E., and Hubbard, E.M. 2007. Inferior colliculus lesions impair eyeblink conditioning in rats. Learn. Mem. 14: $842-846$.

Gabriel, M., Saltwick, S.E., and Miller, J.D. 1975. Conditioning and reversal of short-latency multiple-unit responses in the rabbit medial geniculate nucleus. Science 189: 1108-1109.

Glickstein, M., Cohen, J.L., Dixon, B., Gibson, A., Hollins, M., Labossiere, E., and Robinson, F. 1980. Corticopontine visual projections in macaque monkeys. J. Comp. Neurol. 190: 209-229.

Gould, T.J. and Steinmetz, J.E. 1996. Changes in rabbit cerebellar cortical and interpositus nucleus activity during acquisition, extinction and backward classical conditioning. Neurobiol. Learn. Mem. 65: 17-34.

Halverson, H.E. and Freeman, J.H. 2006. Medial auditory thalamic nuclei are necessary for eyeblink conditioning. Behav. Neurosci. 120: $880-887$.

Hesslow, G., Svensson, P., and Ivarsson, M. 1999. Learned movements elicited by direct stimulation of cerebellar mossy fiber afferents. Neuron 24: 179-185.

Kawamura, K. 1975. The pontine projection from the inferior colliculus in the cat: An experimental anatomical study. Brain Res. 95: 309-322.

Kettner, R.E. and Thompson, R.F. 1985. Cochlear nucleus, inferior colliculus, and medial geniculate responses during the behavioral detection of threshold-level auditory stimuli in the rabbit. J. Acoust. Soc. Am. 77: 2111-2127.

Knowlton, B.J. and Thompson, R.F. 1988. Microinjections of local anesthetic into the pontine nuclei reduce the amplitude of the classically conditioned eyelid response. Physiol. Behav. 43: 855-857.

Knowlton, B.J., Lavond, D.G., and Thompson, R.F. 1988. The effect of lesions of cerebellar cortex on retention of the classically conditioned eyeblink response when stimulation of the lateral reticular nucleus is used as the conditioned stimulus. Behav. Neural Biol. 49: 293-301.

Knowlton, B.J., Thompson, J.K., and Thompson, R.F. 1993. Projections from the auditory cortex to the pontine nuclei in the rabbit. Behav. Brain Res. 56: 23-30.

Kunzle, H. 1975. Autoradiographic tracing of the cerebellar projections from the lateral reticular nucleus in the cat. Exp. Brain Res. 22: $255-266$

Lennartz, R.C. and Weinberger, N.M. 1992. Frequency-specific receptive field plasticity in the medial geniculate body induced by Pavlovian fear conditioning is expressed in the anesthetized brain. Behav. Neurosci. 106: 484-497.

Lewis, L.J., Lo Turco, J.J., and Solomon, P.R. 1987. Lesions of the middle cerebellar peduncle disrupt acquisition and retention of the rabbit's classically conditioned nictitating membrane response. Behav. Neurosci. 101: 151-157.

Love, J.A. and Scott, J.W. 1969. Some response characteristics of cells of the magnocellular division of the medial geniculate body of the cat. Can. J. Physiol. Pharmacol. 47: 881-888.

Mauk, M.D. and Thompson, R.F. 1987. Retention of classically conditioned eyelid responses following acute decerebration. Brain Res. 403: 89-95.

McEchron, M.D., McCabe, P.M., Green, E.J., Llabre, M.M., and Schneiderman, N. 1995. Simultaneous single unit recording in the medial nucleus of the medial geniculate nucleus and amygdaloid central nucleus throughout habituation, acquisition, and extinction of the rabbit's classically conditioned heart rate. Brain Res. 682: $157-166$

Nicholson, D.A. and Freeman Jr., J.H. 2002. Neuronal correlates of conditioned inhibition of the eyeblink response in the anterior interpositus nucleus. Behav. Neurosci. 116: 22-36.

Nowak, A.J., Kehoe, E.J., Macrae, M., and Gormezano, I. 1999. Conditioning and reflex modification of the rabbit nictitating membrane response using electrical stimulation in auditory nuclei. Behav. Brain Res. 105: 189-198.

Oakley, D.A. and Russell, I.S. 1972. Neocortical lesions and Pavlovian conditioning. Physiol. Behav. 8: 915-926.

Oakley, D.A. and Russell, I.S. 1977. Subcortical storage of Pavlovian conditioning in the rabbit. Physiol. Behav. 18: 931-937.

O'Connor, K.N., Allison, T.L., Rosenfield, M.E., and Moore, J.W. 1997. Neural activity in the medial geniculate nucleus during auditory trace conditioning. Exp. Brain Res. 113: 534-556.

Patterson, M.M. 1970. Classical conditioning of the rabbit's (Oryctolagus cuniculus) nictitating membrane response with fluctuating ISI and intracranial CS. J. Comp. Physiol. Psychol. 72: 193-202.

Paxinos, G. and Watson, C. 1998. The rat brain in stereotaxic coordinates. Academic Press, New York.

Poremba, A. and Gabriel, M. 2001. Amygdalar efferents intiate auditory thalamic discriminative training-induced neuronal activity. $J$. Neurosci. 21: 270-278.

Ryugo, D.K. and Weinberger, N.M. 1978. Differential plasticity of morphologically distinct neuron populations in the medial geniculate body of the cat during classical conditioning. Behav. Biol. 22: 275-301.

Schmahmann, J.D. and Pandya, D.N. 1989. Anatomical investigation of projections to the basis pontis from posterior parietal association cortices in rhesus monkey. J. Comp. Neurol. 289: 53-73.

Schmahmann, J.D. and Pandya, D.N. 1993. Prelunate, occipitotemporal, and parahippocampal projections to the basis pontis in rhesus monkey. J. Comp. Neurol. 337: 94-112.

Schmaltz, L. and Theios, J. 1972. Acquisition and extinction of a classically conditioned response in hippocampectomized rabbits (Oryctolagus cuniculus). J. Comp. Physiol. Psychol. 79: 328-333.

Smith, M.C., Coleman, S.R., and Gormezano, I. 1969. Classical conditioning of the rabbit's nictitating membrane response at backward, simultaneous, and forward CS-US intervals. J. Comp. Physiol. 2: 226-231.

Solomon, P.R. and Moore, J.W. 1975. Latent inhibition and stimulus generalization of the classically conditioned nictitating membrane response in rabbits (Oryctolagus cuniculus) following dorsal hippocampal lesions. J. Comp. Physiol. Psych. 89: 1192-1203.

Steinmetz, J.E. 1990. Classical nictitating membrane conditioning in rabbits with varying interstimulus intervals and direct activation of cerebellar mossy fibers as the CS. Behav. Brain Res. 38: 97-108.

Steinmetz, J.E. and Sengelaub, D.R. 1992. Possible conditioned stimulus pathway for classical eyelid conditioning in rabbits. I. Anatomical evidence for direct projections from the pontine nuclei to the cerebellar interpositus nucleus. Behav. Neural Biol. 57: 103-115.

Steinmetz, J.E., Rosen, D.J., Chapman, P.F., Lavond, D.G., and Thompson, R.F. 1986. Classical conditioning of the rabbit eyelid response with a mossy fiber stimulation CS. I. Pontine nuclei and middle cerebellar peduncle stimulation. Behav. Neurosci. 100: $871-880$.

Steinmetz, J.E., Logan, C.G., Rosen, D.J., Thompson, J.K., Lavond, D.G., and Thompson, R.F. 1987. Initial localization of the acoustic conditioned stimulus projection system to the cerebellum essential for classical eyelid conditioning. Proc. Natl. Acad. Sci. 84: 3531-3535.

Supple Jr., W.F. and Kapp, B.S. 1989. Response characteristics of neurons in the medial component of the medial geniculate nucleus during Pavolvian differential fear conditioning in rabbits. Behav. Neurosci. 6: $1276-1286$.

Thompson, R.F. 1976. The search for the engram. Am. Psychol. 31: 209-227.

Thompson, R.F. 1983. Neuronal substrates of simple associative learning: Classical conditioning. Trends Neurosci. 6: 270-275.

Thompson, R.F. 2005. In search of memory traces. Annu. Rev. Psychol. 56: $1-23$.

Winer, J.A., Larue, D.T., and Huang, C.L. 1999. Two systems of giant axon terminals in the cat medial geniculate body: Convergence of cortical and GABAergic inputs. J. Comp. Neurol. 413: 181-197.

Winer, J.A., Diehl, J.J., and Larue, D.T. 2001. Projections of auditory cortex to the medial geniculate body of the cat. J. Comp. Neurol. 430: $27-55$.

Received March 27, 2008; accepted in revised form May 13, 2008. 


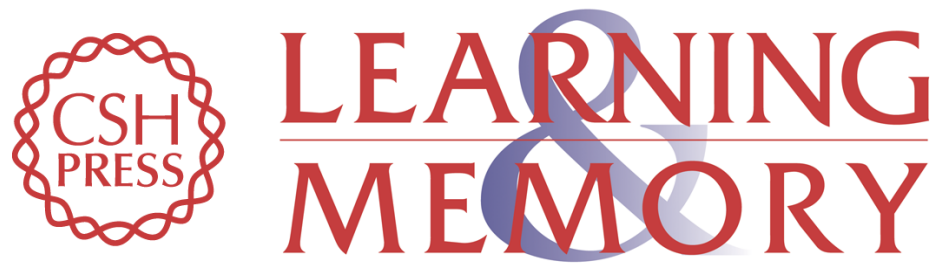

\section{Medial auditory thalamus inactivation prevents acquisition and retention of eyeblink conditioning}

Hunter E. Halverson, Amy Poremba and John H. Freeman

Learn. Mem. 2008, 15:

Access the most recent version at doi:10.1101//m.1002508

References This article cites 51 articles, 8 of which can be accessed free at:

http://learnmem.cshlp.org/content/15/7/532.full.html\#ref-list-1

License

Email Alerting Receive free email alerts when new articles cite this article - sign up in the box at the Service top right corner of the article or click here. 